\title{
Application of PICCO during Anesthesia in Patients Undergoing Transcatheter Ventricular Isolation Plasty Due to Left Ventricular Aneurysm
}

\author{
Yuxuan Zhang, ${ }^{1}$ Quansheng Wang, ${ }^{2}$ Tao Su, ${ }^{1}$ Jing Mei, ${ }^{1}$ Ruixuan Li, ${ }^{1}$ Qiang Yan, ${ }^{1}$ Guiping Xu ${ }^{1}$ \\ ${ }^{1}$ Department of Anesthesia, and ${ }^{2}$ Midong Hospital, People's Hospital of Xinjiang Uygur Autonomous Region, Urumqi, Xinjiang Uygur \\ Autonomous Region, China
}

\section{ABSTRACT}

Introduction: Left ventricular aneurysm (LVA) is a common complication of myocardial infarction. Percutaneous ventricular restoration (PVR) is a new and minimally invasive surgical treatment for LVA. Due to PVR-related complications and high demand on the surgeon, appropriate anesthetic management during PVR is critical for its successful implementation.

Case Presentation: During anesthesia, Pulse index Continuous Cardiac Output (PICCO) not only monitors general hemodynamic parameters but also displays parameters such as cardiac output, myocardial contractility, and pre-cardiac load, providing critical guidance for anesthetic procedures.

Conclusion: This study aimed to explore the application of PICCO during anesthesia by analyzing the clinical anesthetic management of three patients undergoing transcatheter PVR for LVA.

\section{INTRODUCTION}

Necrotic myocardium in patients with myocardial infarction (MI) forms fibrous scar tissue, leading to ventricular wall thinning and outward bulging. Consequently, ventricular aneurysm occurs, generally in the left ventricle, and is known as left ventricular aneurysm (LVA) [Henry 2014]. LVA is associated with a poor clinical outcome [Castelvecchio and Menicanti 2013] and has an incidence of $10 \%$ to $35 \%$ in post-MI patients.

Percutaneous ventricular restoration (PVR) is a surgical procedure for the treatment of LVA [Oliveira 2014]. It is performed as follows: left ventricular (LV) angiography is carried out in patients with LVA and heart failure, followed by right femoral artery puncture for catheterization and installation of the isolation device. As an interventional treatment, this procedure can increase the LV ejection volume, change the ventricular structure to restore $L V$ shape, improve cardiac

Received August 1, 2018; received in revised form November 27, 2018; accepted December 21, 2018.

Correspondence: Guiping Xu, Department of Anesthesia, People's Hospital of Xinjiang Uygur Autonomous Region, 91 Tianchi Road, Tianshan District, Urumqi, 830001, Xinjiang Uygur Autonomous Region, China; +869918563789; fax: +86-9918563789 (e-mail: cnguipingxu@126.com). function, and reduce clinical symptoms [Ige 2015] by isolating the infarcted and aneurysmal LV apex from the remainder of the cavity [Dhakal 2017]. Anesthesia plays an important role throughout the procedure [Evora 2014]. Pulse index Continuous Cardiac Output (PICCO) during anesthesia enables immediate monitoring of intraoperative hemodynamics and other parameters, thereby providing effective guidance for the anesthetic procedure [Boyle 2007].

PICCO is a monitoring technique that combines the transpulmonary thermodilution technique with analysis of the area under the curve of the arterial pressure waveform [Brogly 2016]. It is accurate, bedside-oriented, easy to perform, and has a low risk of injury. PICCO dynamically monitors indicators and provides timely feedback throughout the operation. This facilitates adjustments of the operation plan and medication administration during the peri-anesthesia period, ensuring patient stability and surgical safety [Litton 2012]. We retrospectively analyzed the application of PICCO and its outcomes during anesthetic management of three patients in our hospital who underwent PVR due to LVA.

\section{CASE REPORTS}

This study was conducted in accordance with the Declaration of Helsinki. This study was conducted with approval from the Ethics Committee of the People's Hospital of the Xinjiang Uygur Autonomous Region. Written informed consent was obtained from all participants. Baseline hemodynamic data and patient characteristics are shown in Tables 1 and 2, respectively.

\section{Case 1}

A 53-year-old male patient who had previously undergone coronary artery bypass surgery returned to the hospital because of repeated episodes of chest tightness and shortness of breath after walking about $300 \mathrm{~m}$. The patient was diagnosed with obsolete anterior myocardial infarction, left ventricular aneurysm formation after coronary artery bypass grafting and cardiac function grade II. Echocardiography showed segmental left ventricular wall motion abnormalities, left ventricular apex aneurysm, left ventricular enlargement, a small amount of mitral regurgitation, grade IV ventricular wall motion, and an ejection fraction (EF) of $57 \%$.

Emergency surgery was performed; under local anesthesia, the peripheral vein was opened and a PICCO-specific arterial catheter was placed. A central venous catheter was placed in 
Table 1. Baseline of Preoperative Hemodynamic Data

\begin{tabular}{lccccccc}
\hline & $\mathrm{HR}(\mathrm{bpm})$ & $\mathrm{ELWI}(\mathrm{mL} / \mathrm{kg})$ & $\mathrm{CVP}(\mathrm{cmH} 2 \mathrm{O})$ & $\mathrm{Cl}\left(\mathrm{L} / \mathrm{min} / \mathrm{m}^{2}\right)$ & $\mathrm{SVRI}\left(\mathrm{DS} \cdot \mathrm{m}^{2} / \mathrm{cm}^{5}\right)$ & $\mathrm{SVV}(\%)$ & $\mathrm{MAP}(\mathrm{mmHg})$ \\
\hline Case 1 & 60 & 7 & 8 & 2.69 & 1000 & 10 \\
Case 2 & 70 & 5 & 11 & 2.21 & 2149 & 60 \\
Case 3 & 55 & 10 & 15 & 1.72 & 4317 & 65 \\
\hline
\end{tabular}

HR indicates heart rate; ELWI, extravascular lung water index; CVP; central venous pressure; Cl, cardiac index; SVRI, systemic vascular resistance index; SVV, stroke volume variation; MAP, mean artery pressure.

Table 2. Baseline of Preoperative Diseases

\begin{tabular}{ccccccc}
\hline & Sex & Age & LVEF, \% & Ventricular wall motion classification & NYHA degree & Preoperative diagnosis \\
\hline Case 1 & Male & 53 & 57 & IV & IV & Myocardial infarction of anterior wall with ventricular aneurysm \\
Case 2 & Male & 60 & 50 & IV & IV & Left ventricular apical aneurysm \\
Case 3 & Male & 54 & 41 & Ventricular septal rupture with ventricular aneurysm
\end{tabular}

the jugular vein and connected to the PICCO detector via a transducer. Intraoperative PICCO indicators were measured and adjusted according to cardiac function status. After induction of anesthesia, heart rate (HR) was 60 beats/min and invasive blood pressure (IBP) $80 / 55 \mathrm{mmHg}$. PICCO monitoring showed a systemic vascular resistance index (SVRI) of 1000 dyn $\cdot \mathrm{s} \cdot \mathrm{m}^{2} / \mathrm{cm}^{5}$, cardiac index (CI) of $2.69 \mathrm{~L} / \mathrm{min} / \mathrm{m}^{2}$, stroke volume variation (SVV) of $10 \%$, and pulse pressure variation (PPV) of $9 \%$, indicating that the systemic vascular resistance was reduced. A small dose of the vasoactive drug norepinephrine was administered to maintain circulatory system stability. Continuous pump infusion of lidocaine $(1-2 \mathrm{mg} / \mathrm{kg} / \mathrm{h})$ was used to reduce ventricular stress. Esmolol (10-20 mg) was used for heart rate adjustment. Normal blood gas and electrolyte levels were maintained. The patient returned to the Cardiology Care Unit once he regained consciousness and after the endotracheal tube was removed.

\section{Case 2}

A 60-year-old male patient who had previously undergone percutaneous coronary intervention (PCI) and had one stent in the anterior descending branch presented with chest tightness, shortness of breath, palpitations, and discomfort, but without recent chest pain. The patient was diagnosed with obsolete anterior myocardial infarction with ventricular aneurysm, left ventricular dysfunction, and grade II heart function. Echocardiography showed abnormal segmental left ventricular wall motion, left ventricular apex aneurysm, a slightly enlarged left ventricle, a slightly widened aortic sinus, a small amount of mitral regurgitation, grade IV wall motion, and an EF of 50\%. Consequently, elective PVR for the LVA was performed.

Under local anesthesia, a PICCO catheter was inserted, and a central venous catheter was placed into the right jugular vein and connected to the PICCO detector via a transducer. Mean artery pressure (MAP), CI, central venous pressure (CVP), intrathoracic blood index (ITBI), intrathoracic blood volume (ITBV), extravascular lung water (EVLW), SVV, GFI, HR, and SVRI were recorded. The patient's HR was 70 beats/min; IBP $88 / 52 \mathrm{mmHg}$; CVP 5 cmH $\mathrm{O}_{2}$ SVV 15\%; PPV 17\%; CI $2.21 \mathrm{~L} / \mathrm{min} / \mathrm{m}^{2}$; and SVRI 2149 dyn.s. $\mathrm{m}^{2} / \mathrm{cm}^{5}$, indicating insufficient cardiac capacity. After infusion, the HR was 61 beats/min; IBP 103/68 mmHg; CVP 8 cmH 2 ; SVV 12\%; PPV 12\%; CI $2.61 \mathrm{~L} / \mathrm{min} / \mathrm{m}^{2}$; and SVRI $1997 \mathrm{dyn} \cdot \mathrm{s} \cdot \mathrm{m}^{2} / \mathrm{cm}^{5}$. The surgery was completed without incident and the patient returned to the Cardiology Care Unit after extubation.

\section{Case 3}

A 54-year-old male patient received emergency treatment because of sudden chest pain, chest discomfort and aggravation for two hours. His condition worsened and he was transferred to our hospital. He was diagnosed with chronic heart failure acute attack, cardiac function grade IV, old myocardial infarction, coronary atherosclerotic heart disease, ventricular septal perforation after acute myocardial infarction, ventricular aneurysm and hypertension. PCI intervention was performed and a stent was implanted into the left anterior descending artery. Ventricular septal defect closure with umbrella devices was later performed. At this time, the echocardiography showed abnormal segmental left ventricular wall motion, left ventricular apical aneurysm, ventricular septal perforation, left-to-right shunt at the ventricular level, an enlarged left ventricle, a mildly enlarged right ventricle, mild mitral regurgitation, moderate tricuspid regurgitation, moderate pulmonary hypertension, ventricular wall motion grade IV, and an EF of $41 \%$. Elective PVR was carried out.

In the operating room, the peripheral vein was opened under local anesthesia and a PICCO catheter was placed. PICCO monitoring showed that the HR was $55 / \mathrm{min}$; IBP 156/92 mmHg; CVP $9 \mathrm{cmH}_{2} \mathrm{O}$; SVV 6\%; PPV 7\%; CI $1.72 \mathrm{~L} / \mathrm{min} / \mathrm{m}^{2}$; and SVRI 4317 dyn.s. ${ }^{2} / \mathrm{cm}^{5}$, which 
indicated that the maintenance of $\mathrm{BP}$ depended on high peripheral resistance. Sevoflurane combined with remifentanil was used to maintain anesthesia because sevoflurane also has a vasodilatory effect on peripheral vessels. With continuous inhalation of sevoflurane, the SVRI gradually decreased from 4317 to $2251 \mathrm{dyn} \cdot \mathrm{s} \cdot \mathrm{m}^{2} / \mathrm{cm}^{5}$. The CI increased from 1.72 to $1.97 \mathrm{~L} / \mathrm{min} / \mathrm{m}^{2}$, but IBP dropped to $99 / 60 \mathrm{mmHg}$. A continuous infusion of dopamine $2 \mu \mathrm{g} / \mathrm{kg} / \mathrm{min}$ was administered. The CI gradually increased from 1.97 to $2.33 \mathrm{~L} / \mathrm{min} / \mathrm{m}^{2}$, and $\mathrm{BP}$ increased to $128 / 72 \mathrm{mmHg}$, indicating that the vital signs were stable. As PICCO monitoring showed further improvement in cardiac function (SVRI $2484 \mathrm{dyn} \cdot \mathrm{s} \cdot \mathrm{m}^{2} / \mathrm{cm}^{5}$; CI 2.90 $\mathrm{L} / \mathrm{min} / \mathrm{m}^{2}$; HR 63 beats/min; and IBP $153 / 81 \mathrm{mmHg}$ ), the infusion of dopamine was withdrawn, and the stability of the circulatory system was maintained. After surgery, the patient returned to the Cardiology Care Unit.

\section{DISCUSSION}

Measurement of the precise depth of anesthesia and fine adjustment of circulatory system indices are critical to guarantee steady hemodynamics and increased oxygen supply during anesthetic management while performing PVR. Rational use of cardiovascular drugs during the surgery can prevent and reduce the incidence of myocardial ischemia [O'Donoghue 2011]. During PVR, observation of clinical symptoms or use of conventional monitoring methods result in inaccuracies in determining the timing of the selection of various functional interventions and liquid replenishment [Huang 2014]. PICCO monitoring not only records conventional hemodynamic parameters [Litton 2012], but also measures MAP, CI, ITBI, ITBV, EVLW, SVV, GFI, HR, SVRI, and other indicators [Tagami 2012], allowing for more accurate and timely control of cardiac function and blood volume [Xie 2015].

For most patients with LVA, preoperative echocardiography and physical examinations cannot provide a complete and accurate assessment of cardiac status due to poor preoperative cardiac function [Chen 2012]. PICCO monitoring allows for a more individualized approach in the use of narcotic drugs and limits the impact of inappropriate drugs, thereby achieving stable hemodynamics and a smooth and safe operation.

In conclusion, continuous measurement of SVV, CI, CO, and SVRI using PICCO can accurately reflect the effects of treatment and facilitate targeted intervention through feedback information that can correct intraoperative adverse conditions and functional performance during PVR for LVA.
REFERENCES

Boyle M, Murgo M, Lawrence J, et al. 2007. Assessment of the accuracy of continuous cardiac output and pulse contour cardiac output in tracking cardiac index changes induced by volume load. Aust Crit Care 20:106-12

Brogly N, Schiraldi R, Puertas L, et al. 2016. Pulse contour analysis calibrated by Trans-pulmonar thermodilution (Picco Plus) for the perioperative management of a caesarean section in a patient with severe cardiomyopathy. Braz J Anesthesiol 66:329-32.

Castelvecchio S, Menicanti L. 2013. Left ventricular reconstruction: update to left ventricular aneurysm/reshaping techniques. Multimed Man Cardiothorac Surg 2013:mmt002.

Chen X, Qiu ZB, Xu M, et al. 2012. Surgery for left ventricular aneurysm after myocardial infarction: techniques selection and results assessment. Chin Med J (Engl) 125:4373-9.

Dhakal BP, Oliveira GH. 2017. Percutaneous Ventricular Restoration with a partitioning device for ischemic heart failure treatment. Curr Heart Fail Rep 14:87-99.

Evora PR, Tubino PV, Gali LG, et al. 2014. A variant technique for the surgical treatment of left ventricular aneurysms. Rev Bras Cir Cardiovasc 29:645-9.

Henry MJ, Preventza O, Cooley DA, et al. J 2014. Left ventricular aneurysm repair with use of a bovine pericardial patch. Tex Heart Inst 41:407-10.

Huang XS, Gu CX, Yang JF, et al. 2014. A pilot study of systolic dyssynchrony index by real-time three-dimensional echocardiography predicting clinical outcomes to surgical ventricular reconstruction in patients with left ventricular aneurysm. Interact Cardiovasc Thorac Surg 19:938-45.

Ige M, Al-Kindi SG, Attizzani G, et al. 2015. Percutaneous left ventricular restoration. Heart Fail Clin 11:261-73.

Litton E, Morgan M. 2012. The PiCCO monitor: a review. Anaesth Intensive Care 40:393-409.

O'Donoghue RD, Duncan AE, Fraser TG, et al. 2011. An echocardiographic evaluation of valvular function and ventricular patch repair during surgical exclusion of left ventricular pseudoaneurysm. Anesth Analg 113:713-6.

Oliveira GH, Al-Kindi SG, Bezerra HG, et al. 2014. Left ventricular restoration devices. J Cardiovasc Transl Res 7:282-91.

Tagami T, Kushimoto S, Tosa R, et al. 2012. The precision of PiCCO measurements in hypothermic post-cardiac arrest patients. Anaesthesia $67: 236-43$

Xie H, Sun H, Li Z, et al. 2015. Successful fluid resuscitation in a patient with high extravascular lung water index by restricted fluid infusion strategy under pulse indicator continuous cardiac output monitoring. Am J Emerg Med 33:739.e1-4. 